\title{
Influence of GSM900 electromagnetic fields on the metabolic rate in rodents
}

\author{
A. El Ouardi ${ }^{1}$, J. Streckert ${ }^{1}$, A. Lerchl ${ }^{2}$, K. Schwarzpaul ${ }^{2}$, and V. Hansen ${ }^{1}$ \\ ${ }^{1}$ Chair of Electromagnetic Theory, University of Wuppertal, 42097 Wuppertal, Germany \\ ${ }^{2}$ School of Engineering and Science, Jacobs University Bremen gGmbH, 28759 Bremen, Germany
}

\begin{abstract}
The development of exposure devices for investigating possible effects of mobile communication systems to non-restrained animals aims at a homogenous field distribution in the area the animals occupy. In the presented $900 \mathrm{MHz}$ exposure device a quite good field homogeneity of $5 \%$ (including the standing wave contribution due to internal reflections) is reached in the cage region mainly by flattening the transverse field. For the standard waveguide (WR1150) without dielectric sheets this value reads $14 \%$. The desired maximal whole body specific absorption rate (SAR) of $4 \mathrm{~W} / \mathrm{kg}$ in the Djungarian hamster model is achieved at an input power of only $3.7 \mathrm{~W}$.
\end{abstract}

\section{Introduction}

Several experiments were performed in the last few years in order to investigate possible biological effects of radio frequency (rf) signals in the non-thermal range, i.e. with a maximum rf-induced temperature increase fairly below $1^{\circ} \mathrm{C}$. Some published results in the frequency region of mobile communication signals indicating body weight alterations might support the hypothesis that rf signals can influence the metabolic rate of rodents. Therefore, an experiment was set up by the Federal Office for Radiation Protection within the framework of the German Mobile Telecommunication Research Programme (BfS) to confirm or disapprove this thesis. This paper presents details of the engineering part of the project.

Four plastic cages (dimensions (width $\times$ height $\times$ length) in $\mathrm{mm}$ : $147 \times 117 \times 355$ ), each housing up to three hamsters for one week, are exposed to a GSM test signal at $900 \mathrm{MHz}$. Air of defined speed $(30-401 / \mathrm{h})$ flows through the cage. In

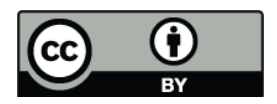

Correspondence to: V. Hansen (hansen@uni-wuppertal.de)

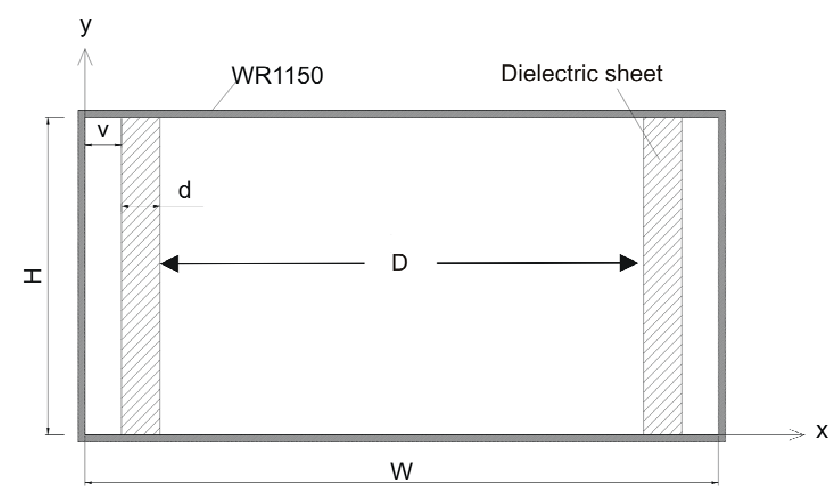

Fig. 1. Cross section of waveguide WR1150 with inserted dielectric sheets.

the supplied air the temperature and the concentration of oxygen and carbon dioxide is measured. The analysis of the data gives information about the metabolism of the animals. Due to the rf field whole body SAR values of $4 \mathrm{~W} / \mathrm{kg}, 0.4 \mathrm{~W} / \mathrm{kg}$, and $0.08 \mathrm{~W} / \mathrm{kg}$ are applied added by sham exposure phases $(0 \mathrm{~W} / \mathrm{kg})$. The choice of the SAR is done randomly and in a blinded manner.

\section{Exposure device}

The field distribution in the volume of a cage should be homogeneous in order to obtain uniform exposure conditions for the animals regardless of their location. This can be achieved by inserting each cage into a rectangular waveguide (type WR1150: internal dimensions $\mathrm{W}=292.1 \mathrm{~mm}$, $\mathrm{H}=146.05 \mathrm{~mm})$ equipped with additional inner dielectric sheets (Fig. 1). 


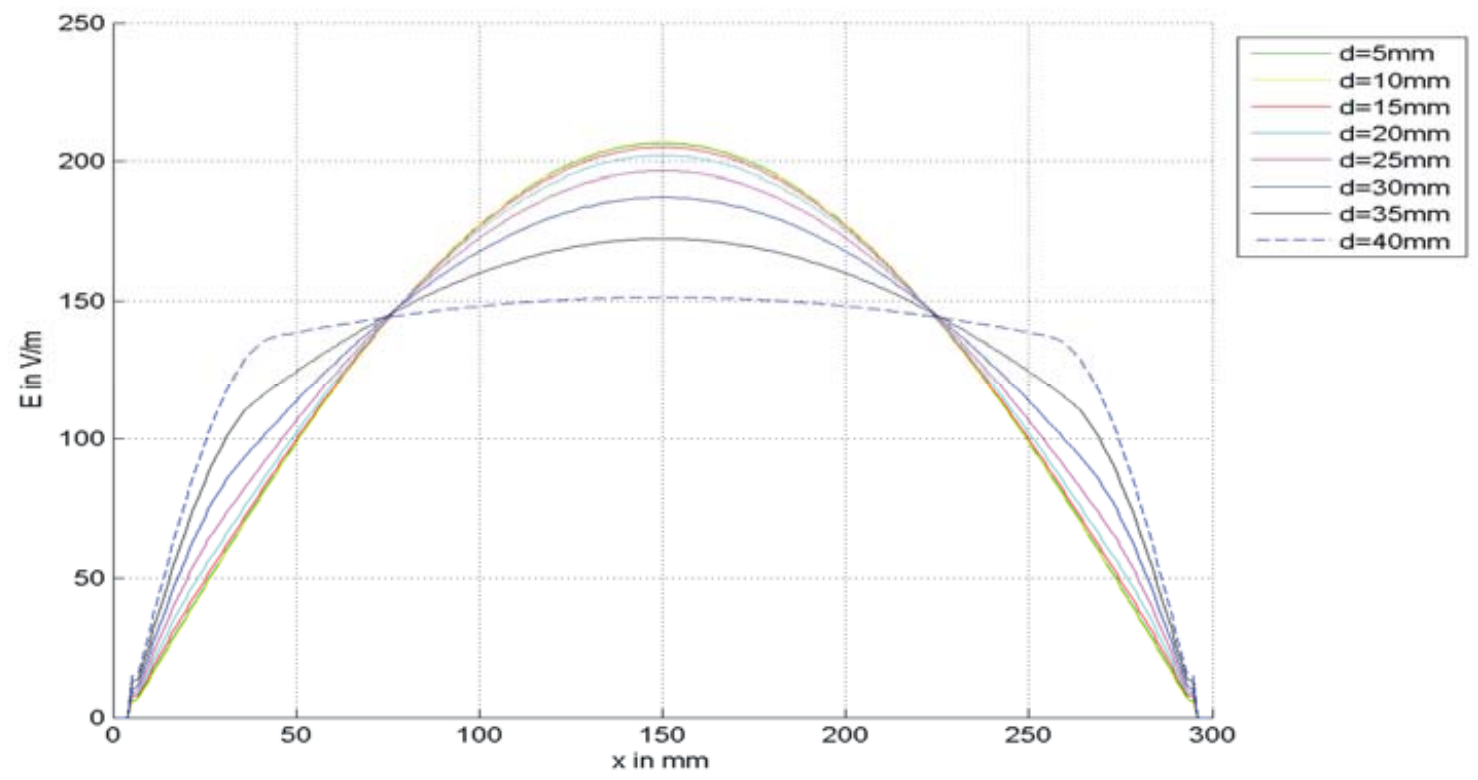

Fig. 2. Field distribution in transverse direction for $v=0, \varepsilon_{r}=5$, and different sheet widths $d(P=1 \mathrm{~W})$.

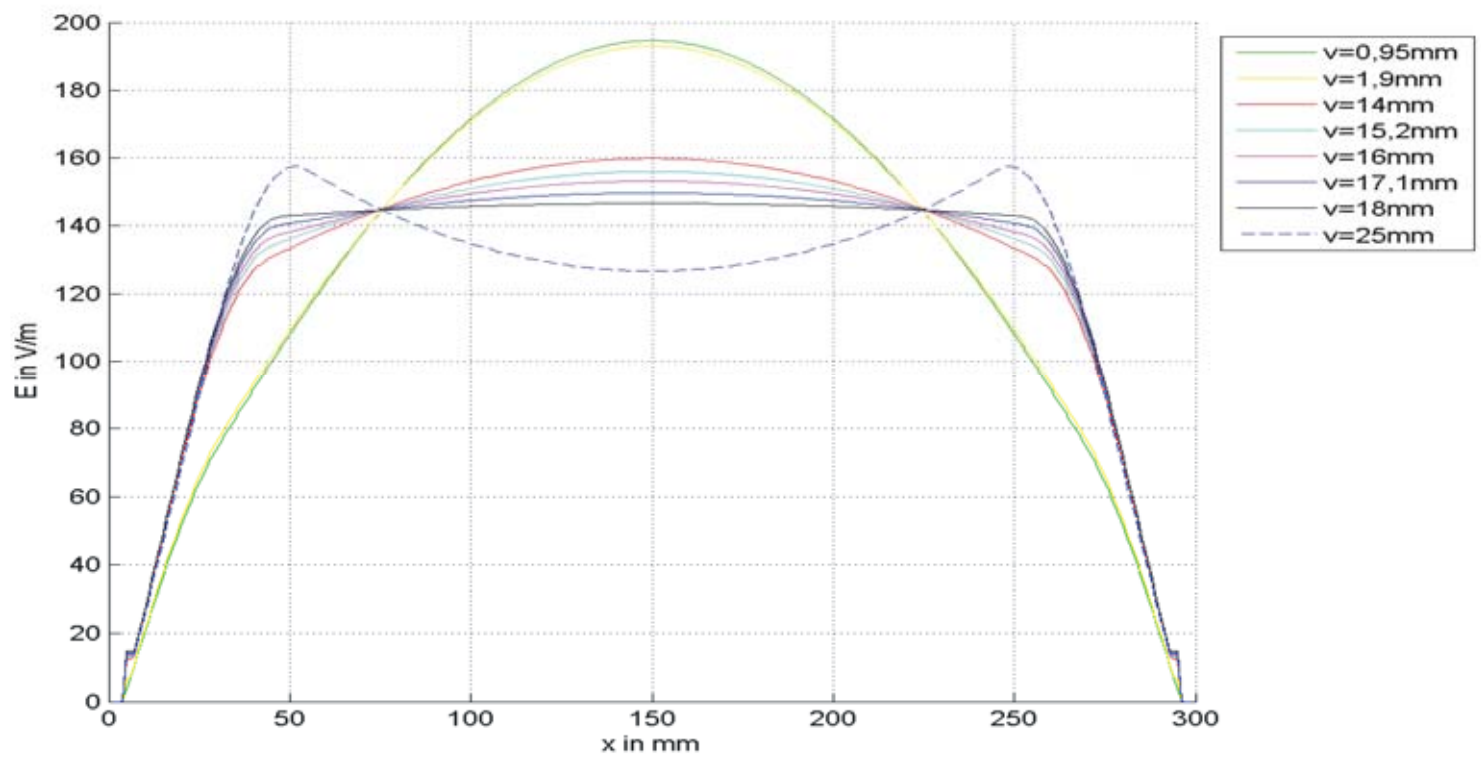

Fig. 3. Field distribution in transverse direction for $d=25.4 \dot{\mathrm{mm}}, \varepsilon_{r}=5$, and different distances $v(P=1 \mathrm{~W})$.

\subsection{Concept}

In a rectangular waveguide with standard dimensions for the carrier frequency of $900 \mathrm{MHz}$ only the fundamental $\mathrm{H}_{10^{-}}$ wave can propagate. However, it has a sinusoidal transverse field. In order to flatten the field, two dielectric sheets of thickness $d$, distance $D$ and height $H$ are positioned symmetrically to the waveguide axis. In principal, by use of matters in waveguides the propagation of higher-order wave types can be provoked, because the dielectric material re- duces their cut-off frequencies. This is also the case in the structure shown in Fig. 1. To overcome this drawback, the fundamental mode is excited selectively by using a transition from the empty metallic standard waveguide to the optimized structure with dielectric sheets. Both measures - flattening of the field and mode-selective excitation - are considered in the following. 


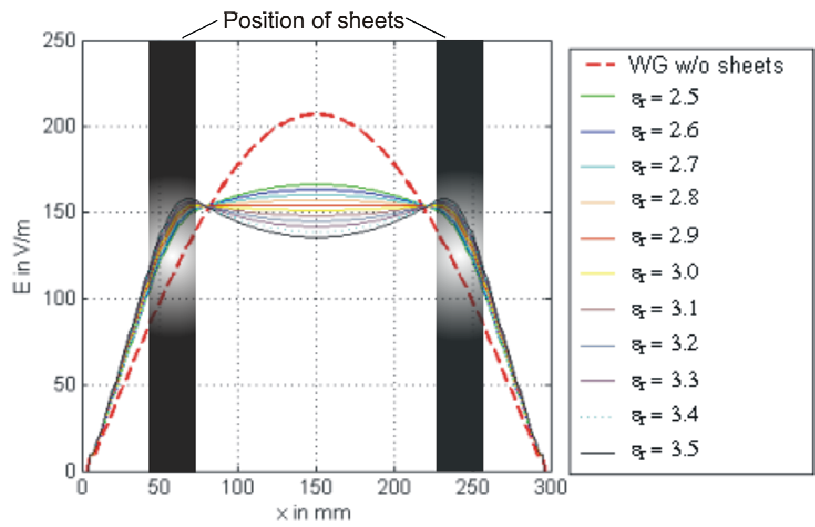

Fig. 4. Field distribution in transverse direction for $d=30 \mathrm{~mm}$, $v=38.5 \mathrm{~mm}$, and variable permittivity $\varepsilon_{r}(P=1 \mathrm{~W})$.

\subsubsection{Improved field homogeneity}

Due to the width of the cage including its cover, the distance $D$ must not be smaller than $155 \mathrm{~mm}$. Material characteristics, thickness and position of the sheets are modified to achieve a good field homogeneity between those walls. For optimising the dielectric sheets different points are discussed.

First, with the permittivity $\varepsilon_{r}=5$ and the distance $v=0 \mathrm{~mm}$ (i.e. the dielectric sheets are in contact with the interior side of the metal walls of the waveguide) the thickness $d$ is varied in order to find the optimum thickness for a homogeneous field distribution in the area between both dielectric sheets. The calculation of the field distribution is performed with help of the software package MICROWAVE STUDIO $^{\mathrm{TM}}$ (CST, 2006). This software has a graphical user interface based on Windows and the calculation uses the FI method (Finite Integration technique (Weiland, 1990)). For an increasing thickness $d$ the electric field distribution of the fundamental mode becomes more and more homogeneous in transverse direction reaching a rather constant field for $d=40 \mathrm{~mm}$ (Fig. 2), but for $d>20 \mathrm{~mm}$ also higher-order waves become propagable.

Another possibility is to keep the thickness $\mathrm{d}$ constant and to increase the distance $v(v>0)$. In Fig. 3 the field distribution in transversal direction is plotted for different distances $v$, for $d=1^{\prime \prime}=25.4 \mathrm{~mm}$ and for the permittivity $\varepsilon_{r}=5$ as before. This solution seems better than the first, because the field is quite homogeneous at $v_{\mathrm{opt}}=18 \mathrm{~mm}$ with less material usage.

The last point concerns the reduction of the permittivity of the dielectric sheets with the aim to use standard material available on the market at moderate costs. For this investigation the thickness $\mathrm{d}$ and the position $\mathrm{v}$ of the dielectric sheets are fixed, while $\varepsilon_{r}$ is varied. From the transverse electrical field distribution shown in Fig. 4 for an input power of $1 \mathrm{~W}$, $d=30 \mathrm{~mm}$, and $v=38.5 \mathrm{~mm}$ the permittivity $\varepsilon_{r}=2.9$ is found to be the best solution for a homogeneous field distribution. With these parameters a quasi TEM waveguide can be designed.

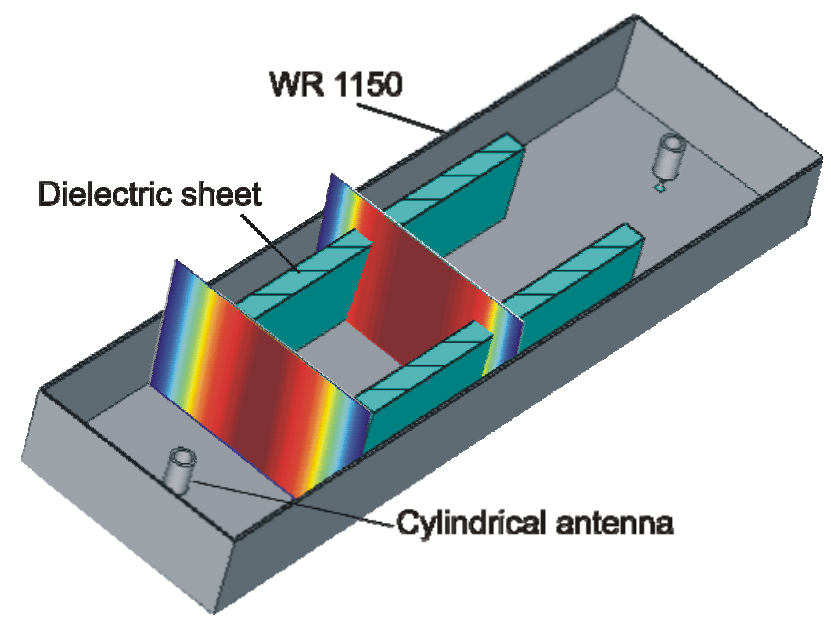

Fig. 5. Field distribution in two cross-sections of the total configuration.

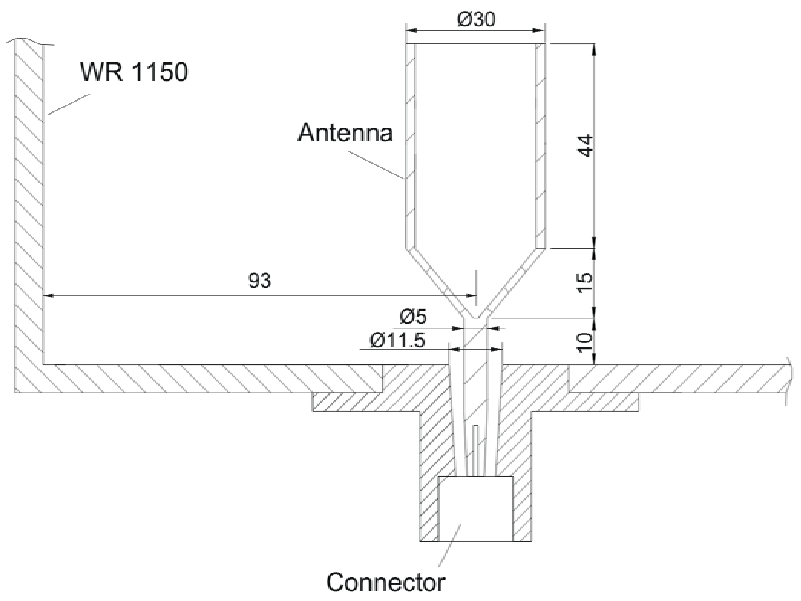

Fig. 6. Longitudinal cut through the coaxial-to waveguide adapter for excitation of the fundamental mode, optimized for $900 \mathrm{MHz}$.

\subsubsection{Selective wave excitation}

For the opimization given above, the first-order higher mode $\left(\mathrm{H}_{20}\right)$ is able to propagate in addition to the fundamental mode, but only the propagation of the fundamental mode is desired. This can be achieved by using a section of standard waveguide with an integrated coaxial-to-waveguide adapter in front of the waveguide section with the dielectric sheets (Fig. 5). From cross-sectional cuts of the calculated field distribution, like the two plotted in Fig. 5, it turns out that

1. in a distance of $160 \mathrm{~mm}$ from the exciting cylindrical antenna, whose dimensions are given in Fig. 6, the standard waveguide propagates solely the $\mathrm{H}_{10}$-mode (with the sinusoidal transverse field) yielding a good excitation efficiency for the fundamental mode at the transition to the waveguide section with dielectric sheets, 


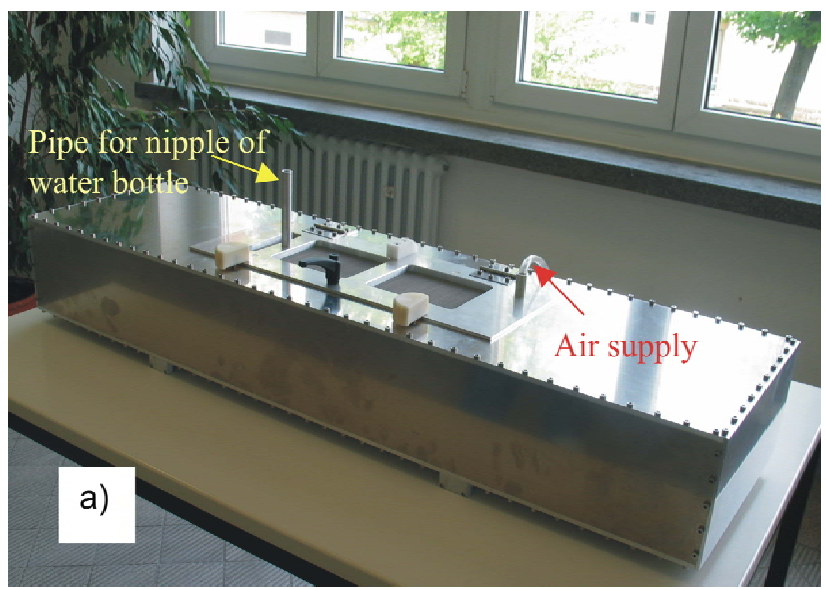

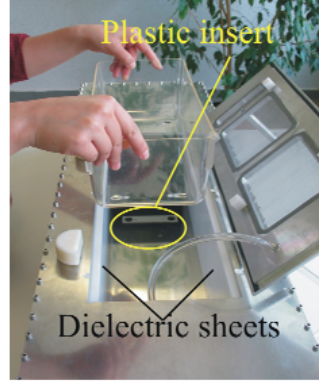

b)

Fig. 7. Built-up rectangular waveguide (a) with dielectric sheets (b).

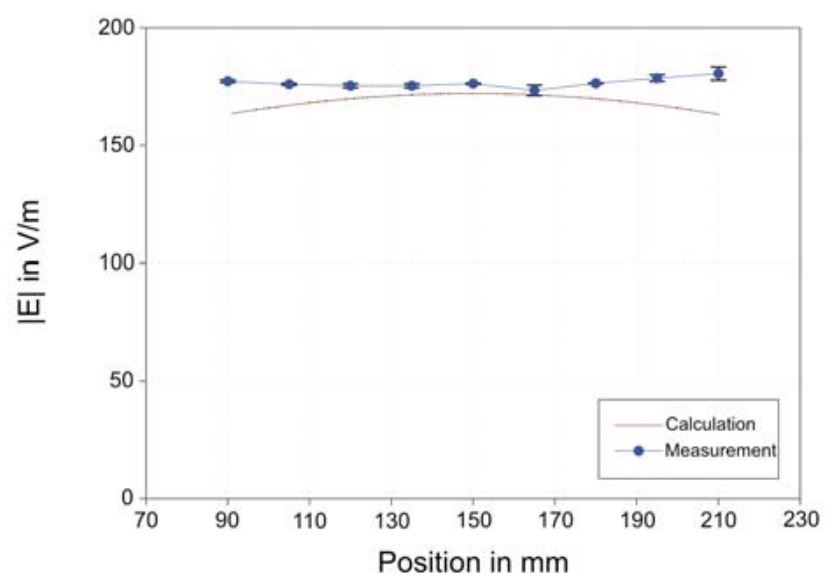

Fig. 8. Comparison of measured and calculated transverse field distribution between the dielectric sheets for $d=30 \mathrm{~mm}, v=38.5 \mathrm{~mm}$, and $\varepsilon_{r}=2.5\left(P_{i n}=1 \mathrm{~W}\right)$.

while the overlap integral between $\mathrm{H}_{10^{-}}$and $\mathrm{H}_{20}$-mode gives zero, and

2. in a distance of $72.5 \mathrm{~mm}$ from the beginning of the dielectric sheets a very good homogeneity of the field between both sheets (area of the cage) is obtained.

It should be mentioned that for reasons of comparison explained below, the field distributions in Fig. 5 are not presented for sheets with the optimum permittivity of 2.9 (leading to a perfectly homogeneous transverse field between the sheets) but for $\varepsilon_{r}=2.5$.

The waveguide is designed symmetrically, i.e. a second coaxial-to-waveguide adapter is placed behind the section with the dielectric sheets.

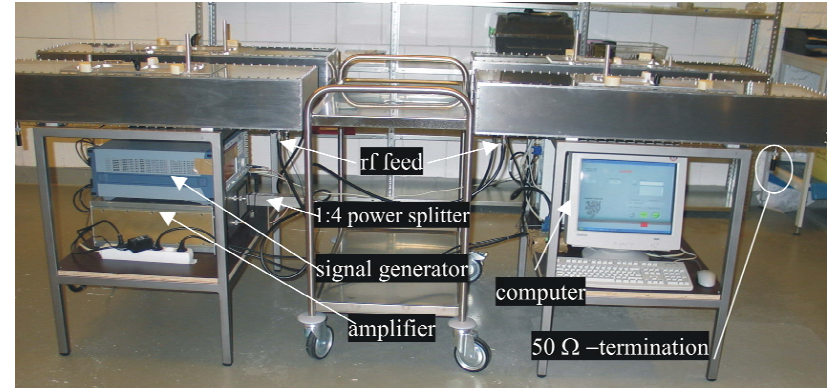

Fig. 9. Photograph of the entire installed rf exposure set-up.

\subsection{Results}

Figure 7a shows a photograph of the built-up rectangular waveguide. The total length is $1.1 \mathrm{~m}$, the width $30 \mathrm{~cm}$ and the height about $18 \mathrm{~cm}$. The top plate is closed by a hinged lid with a fine metal grid for reasons of lighting and simultaneous electromagnetic shielding. The pipe on the left is used for inserting the nipple of the water bottle, the second pipe on the right is needed as a feed-through for the flexible tube of the air supply. These pipes are dimensioned as circular waveguides-beyond-cut-off, thus being open or shut from an mechanical or respectively an electromagnetical point of view. Figure $7 \mathrm{~b}$ shows the waveguide with the lid opened. The dielectric sheets and one of the plastic inserts for positioning the cage at a defined position are visible.

Using a simulation model of the waveguide including the coax-to-waveguide adapters, the dielectric sheets, and a $50 \Omega$ termination at the output port, a total reflexion coefficient of approx. $-20 \mathrm{~dB}$ is calculated at the input port. This value is confirmed by S-parameter measurements with the help of a network analyzer.

Since the permittivity measurement of the actual dielectric sheets yielded a value of 2.5 , deviating from the optimum 
value of 2.9, the former was used for the field computations. Moreover, measurements of the field within the waveguide were performed with the help of a monopole probe inserted through bores in an adapter plate covering the opening in the top side of the waveguide. Figure 8 shows the comparison between the measured and calculated transverse field distribution for an input power of $1 \mathrm{~W}$. The agreement within $9 \%$ is rather satisfying, particularly since an uncertainty of the permittivity measurement of $5 \%$ must be considered.

Figure 9 shows the entire exposure system installed at the Jacobs University Bremen. It consists of four rectangular waveguides each housing one cage, of a signal generator/modulator/amplifier-combination and of a 1:4power splitter in order to distribute the output power to the four waveguides. $50 \Omega$ terminations are mounted to the output connectors of the waveguides. The computer is used to select the power level (and thereby the applied SAR) and to record a control signal from a rf detector-diode. This signal is permanently analysed with the view to check the functionality of the equipment.

The determination of the field- and SAR-distribution in case of the waveguides filled with animals is only possible by numerical computations. A FDTD (Finite Difference Time Domain) in-house code (Bitz, 2004) was used involving a dielectric computer model of a hamster developed from ten MRI cut views. 21 different tissues have been considered.

From the analysis of the computations for different configurations and positions of the hamsters within their cages the necessary input power for the desired SAR-values was found.

In order to achieve the maximum whole body SAR of $4 \mathrm{~W} / \mathrm{kg}$ the $\mathrm{rf}$ amplifier has to provide a total average power of $15,6 \mathrm{~W}$ to feed the four waveguides. Comparing this value with the waveguides' total input power of $4 \times 3.7 \mathrm{~W}=14.8 \mathrm{~W}$ indicates that only $0.8 \mathrm{~W}$ are lost by absorption and reflection in the splitter, the cables and connectors. The uniformness of input powers amongst the four waveguides is within $6 \%$.

\section{Conclusions}

A $900 \mathrm{MHz}$ exposure set-up for the investigation of the metabolic rate of rodents was developed at the Bergische Universität Wuppertal and installed at the Jacobs University Bremen. The set-up consists of four rectangular waveguides designed for quasi TEM operation with a nearly homogeneous transverse field distribution due to specially dimensioned dielectrical sheets. The input powers needed for generating the demanded SAR values within the animals were found by means of computer simulations involving a model of the entire waveguide with one cage and up to three hamsters in different arrangements and postures. The detailed dosimetrical results will be presented elsewhere. The modulation signal was chosen according to the generic GSM signal defined in Schüller et al. (2000).

\section{References}

BfS: German Mobile Telecommunication Research Programme, http://www.emf-forschungsprogramm.de, March 2008.

Bitz, A.: Numerische Feldberechnung im biologischen Gewebe: Exposition von Personen, Tieren und isolierten biologischen Systemen in elektromagnetischen Feldern, Dissertation, Bergische Universität Wuppertal, 11-20, 2004.

CST GmbH: MICROWAVE STUDIO ${ }^{\mathrm{TM}}$ Version 2006B, Darmstadt, 2006.

Schüller, M., Streckert, J., Bitz, A., Menzel, K., and Eicher, B.: Proposal for generic GSM test signal, Proc. 22st BEMS Annual Meeting, Munich, Germany, 122-123, 2000.

Weiland, T.: Maxwell's grid equations, Frequenz, 44, 9-16, 1990. 